\title{
History of Two Fundamental Principles of Physics: Least Action and Conservation of Energy
}

\author{
Agamenon R. E. Oliveira \\ Polytechnic School of Rio de Janeiro, Federal University of Rio de Janeiro, Rio de Janeiro, Brazil \\ Email: agamenon.oliveira@globo.com \\ Received 7 January 2014; revised 27 February 2014; accepted 9 March 2014 \\ Copyright (C) 2014 by author and Scientific Research Publishing Inc. \\ This work is licensed under the Creative Commons Attribution International License (CC BY). \\ http://creativecommons.org/licenses/by/4.0/ \\ c) (i) Open Access
}

\begin{abstract}
One of the aims most sought after by physics along the years has been to find a principle, the simplest possible, into which all natural phenomena would fit, and which would also allow the calculation of all past occurrences and principally future occurrences. Evidently, this is far from being reached and quite probably does not even exist. Nevertheless, an approximation to this ideal is always possible and the history of physics shows that some results in this direction have been achieved. Thus, the history of the principles of least action and conservation of energy presented in this paper explains the search for this ideal.
\end{abstract}

\section{Keywords}

History of Physics; Epistemology of Physics; Fundamental Principles of Physics

\section{Introduction}

Among the more general laws which fall within the appointed above perspective is the principle of least action (LAP). In all branches of science to which it has been applied, it is possible to obtain a general explanation of certain characteristics of the phenomena involved, as well as provide the rules which indicate how these phenomena vary in time and space and how to determine them. In this way, the principle of least action has occupied a central position in modern physics, together with the principle of the conservation of energy (Oliveira, 2012). It is worth pointing out that the latter holds a position similar to the former and governs a large number of physical processes.

The principle of the conservation of energy can be deduced from the principle of least action and consequently is contained within it. The reciprocal, however, is not true, which confers a more general character to the principle 
of least action. As an example we can consider the motion of a particle free from the action of any force. According to the principle of the conservation of energy, the particle should move at a constant velocity, but nothing is said about the direction of the velocity vector, since the kinetic energy does not depend on that direction. In principle, the trajectory of the particle could be as much rectilinear as curvilinear, if the motion is considered only from the energy point of view. If we apply the principle of least action, we conclude that the particle must move in a straight line.

This problem is entirely general and can be generalised. In the case of a spherical pendulum, that is, a concentrated mass moving without friction over a fixed spherical surface, the principle of the conservation of energy only states that during the upward movement, the kinetic energy diminishes by a certain quantity and increases during the downward motion. The trajectory of the particle cannot be determined by the conservation of energy, while the principle of least action completely resolves all the questions related to this type of motion.

The fundamental difference in the application of the two principles to any problem is that the principle of the conservation of energy provides only one equation, while it is necessary to obtain as many equations as there are variables or degrees of freedom, in order to completely determine the motion of the system. Thus, in the case of the free particle, three equations are needed and in the case of a spherical pendulum only two, whereas the principle of least action provides, in all cases, as many equations as there are variables.

We should bear in mind that the LAP provides various equations from a single formula, since it deals with a variational principle. Its great advantage is that from an infinite number of imaginable virtual motions, under the particular given conditions, it indicates a well-defined motion by means of a simple criterion and points to the effective motion. This criterion means that for an arbitrary motion infinitely close to the effective motion, or more precisely, for all infinitely small variations of the given motion, consistent with the given conditions of the connection, a certain characteristic function of the variable is annulled. In this way, an equation is deduced from every independent variable, that is, from every degree of freedom of the system, as a problem of maximum or minimum.

\section{The Least Action Principle}

The origins of the LAP go back to Leibniz ${ }^{1}$. In a theorem enunciated by him, it is established that of all the worlds which could be created, the effective world is that which contains, along with all the inevitable bad, the maximum good. Although postulated in the moral terrain, the theorem proposes a variational type solution like the LAP. According to some historians, Leibniz first formulated this principle in a letter dated 1707, whose original had been lost. Later, the LAP would be developed by Pierre-Louis Moreau de Maupertuis (1698-1759), Euler and Lagrange ${ }^{2}$.

The LAP is indissolubly linked to the name of Maupertuis, who not only recognized the existence and significance of this principle, but also used all his influence in the scientific world to work for its acceptance. We cannot forget that he was nominated by Frederick the Great as President of the Prussian Academy of Science.

Maupertuis was born in Saint Malo, France, in 1698, and was one of the great disseminators in France of Newtonian theories, especially that of mechanics and the law of universal gravitation (Newton, 1952). To this end, he conducted an expedition to Lapland, in 1736-37, to measure the acceleration of gravity, with a view to confirming Newton's supposition about the flattening of the earth. The positive results obtained by the expedition strongly influenced the acceptance of Newton's ideas on the European continent.

The LAP was discussed by Maupertuis in his paper entitled Accord des Différentes Lois de la Nature qui Avaient Jusqu'ici Paru Incompatibles presented to the Academy of Science of Paris in 1744. As we know, this principle was originally formulated for geometric optics and was only later extended to the domain of mechanics.

In his paper, Maupertuis enunciates the three laws which light must obey:

1) In a uniform medium light moves in a straight line.

2) When light encounters a medium it cannot penetrate, it is reflected and the angle of refraction is equal to the angle of incidence.

3) When light passes from one transparent medium to another, its trajectory, after meeting the new medium,

${ }^{1}$ Koenig, professor at La Haye, and a member of the Berlin Academy, asserted that Leibniz was the first to formulate the principle of least action. This affirmation was based on a letter from Leibniz to Hermann dated $16^{\text {th }}$ October 1707 , whose authenticity was questioned by several scholars of the matter. See (Gueroult, 1967).

${ }^{2}$ Although this correspondence has never been found, Leibniz pronounced several times on the principle of least action, it is not improbable that he himself was the first to formulate this principle. Maupertuis' defenders, however, argue that he was responsible for the thoroughness and precision of the formulation. See (Gueroult, 1967).

${ }^{3}$ For a detailed exposition of Maupertuis’ principle, as well as the paper cited, see (de Maupertuis, 1744) at p. 99. 
forms an angle with the previous trajectory, so that the sine of the refraction angle is always in the same ratio as the sine of the incidence angle.

The called law of sines for refraction had already been proposed by Descartes and independently by Snell.

Dioptrics is one of Descartes' earliest works. He refers to it by name in a letter to Marin Mersenne (1588-1648) of 25 November 1630. Finally Dioptrics is published together with the Discourse on Method, the Meteorology and the Geometry in the summer of 1637. Pierre Fermat (1601-1665) disagreed with Descartes' arguments and only with experiments by Leon Foucault (1819-1868) and Hippolyte Fizeau (1819-1896), in the middle of century XIXth would Fermat's hypotheses be confirmed. It is important to emphasize the figure of Fermat in the history of LAP. A principle with his name appears in optics context. The original statement of Fermat's principle was: the actual path between two points taken by a beam of light is the one which is traversed in the least time. This principle was used to derive Snell's law in 1657, being the first variational principle enunciated in physics such that Fermat is recognized as a key figure in the historical development of the fundamental LAP in physics.

Let us see then how Maupertuis (de Maupertuis, 1744) formulated the LAP, extracting from the famous paper the part in which the principle appears:

After meditating deeply on this topic, it occurred to me that light, upon passing from one medium to another, has to make a choice, whether to follow the path of shortest distance (the straight line) or the path of least time. But why should it prefer time over space? Light cannot travel both paths at once, yet how does it decide to take one path over another? Rather than taking either of these paths per se, light takes the path that offers a real advantage: light takes the path that minimizes its action. Now I have to define what I mean by "action". When a material body is transported from one point to another, it involves an action that depends on the speed of the body and on the distance it travels. However, the action is neither the speed nor the distance taken separately; rather, it is proportional to the sum of the distances travelled each multiplied by the speed at which they were travelled. Hence, the action increases linearly with the speed of the body and with the distance travelled. This action is the true expense of Nature, which she manages to make as small as possible in the motion of light.

Maupertuis arrived in this way to a problem of minimization. When making this function minimum, he concluded that the sines of the angle of incidence and refraction equals the inverse ratio of the speeds at which light moves in each medium. This result disagrees with that of Fermat and is in agreement with Descartes. This formulation proposed by Maupertuis does not allow conclusions to be drawn regarding the laws which govern the phenomenon nor the conditions which must be met.

Following the path opened by Maupertuis, Euler ${ }^{4}$ extends the ideas underlying the principle of least action and proposed a principle formulated as a mathematical theorem applied to mechanics for the case of constant energy. Euler affirms: Since all natural processes obey certain laws of maximum or minimum, there is no doubt that the curves described by the bodies under the influences of arbitrary forces, also possess some property of maximum and minimum. He further added that the form proposed by his theorem was only applied when the forces depend on the position and that dissipative systems would not lend themselves to a description of this nature.

After Euler (Euler, 1952), it was Lagrange ${ }^{5}$ who expressed the LAP in an entirely general formulation as a principle of stationary action for a general system of $n$ bodies interacting among themselves. This undertaking is made in his Mécanique analytique, published, as we know, in 1788.

In 1834, William Rowan Hamilton (1805-1865) showed that the LAP admits other representations. In this way he established a strong analogy between mechanics and optics, relating Fermat's principle to that of Maupertuis. This was how he arrived at the current and most used form of the LAP as a variational principle of mechanics, known as the Hamilton principle (Dugas, 1988).

Initially, the postulation of the LAP did not provoke any major commotion nor did it have any considerable effect on the advancement of science, even after the general formulation made by Lagrange. The existence of this principle was considered more a mathematical curiosity and even an unnecessary corollary to the laws of Newton. Nor was there any lack of voices raised against its usefulness. In 1837, Poisson called the LAP the worthless rule.

Only with investigations by William Thomson (1824-1907), Peter Guthrie Tait (1831-1901), Gustav Robert Kirchoff (1824-1887), John Von Neumann (1832-1925) and Ludwig Boltzmann (1844-1906), principally, was it proved that the LAP was the most suitable method for resolving problems of hydrodynamics and elasticity. Fur-

\footnotetext{
${ }^{4}$ According to (Moreira, 1998), Lanczos in his “The Variational Principles of Mechanics” affirms that Euler discovered the Principle of Least Action independently of Maupertuis.

${ }^{5}$ As we know, Lagrange in his "Mécanique Analytique”, postulated a general formulation of the Principle of Least Action for a system of interacting $n$ bodies. See (Lagrange, 1989).
} 
thermore, the LAP was unbeatable when the usual methods of mechanics functioned only with difficulty or even failed. It was only then that its worth and reach began to be perceived. This is clear in the following affirmation by Thomas Tait, made in 1867:

Maupertuis's celebrated principle of least action has been, to date, looked upon more as a curiosity and a property of some strange forms of motion than as a useful rule in kinetic investigations. We are strongly impressed and convinced of a much more profound significance attached to it, not only in the abstract dynamic, but also in the theory of the various branches of physical science now beginning to receive a dynamic explanation (Plank, 1993).

As time went by and with the success of its applications, there was a perception of the fundamental significance of LAP as a general principle which could be applied to systems whose internal physical mechanisms were entirely unknown, or so complicated that they could not be represented by means of ordinary systems of coordinates. Another important fact is that after Boltzmann, Rudolf Clausius (1822-1888) perceived the close relationships between the LAP and the second law of thermodynamics (Truesdell, 1980). It is possible to derive the LAP from the law of maximum entropy. Furthermore, Hermann von Helmholtz (1821-1894), for the first time, demonstrates the existence of a complete systematic application of the LAP to the three great branches of physics: mechanics, electrodynamics and thermodynamics. This gave the LAP a higher status and deepened comprehension of it as a general principle.

In this way, the LAP followed a course similar to the principle of the conservation of energy which, as we know, was originally considered a mechanical principle, despite its general validity.

More recently, Joseph Larmour (1857-1942) in 1900 and Karl Schwarzchild (1873-1916) in 1903, among others, deduced the fundamental equations of electrodynamics and of the theory of the electron from the Hamilton principle.

The LAP also demonstrated its validity and explicative potential, with regard to quantum and relativistic mechanics. Initially, the probabilistic characteristic of quantum mechanics appeared to exclude the LAP from its field of application. However, Feynman, in a classic study, showed that it was possible to include it in this field and created a variational principle embodying the quantum phenomena.

Finally, one of the most brilliant results attained by the LAP was the fact that Einstein's theory of relativity had shown that it occupied an outstanding position among the laws of physics. This is because the function denominated action, according to Hamilton, though not that of Maupertuis, is an invariant with regard to all the transformations of Hendrik Lorenz (1853-1928), signifying that it is independent of the observers' system of reference. From this brief historical outline of the LAP, everything indicates that it appears to govern all of nature's reversible processes. What remains open is that it offers no explanation for the phenomenon of irreversibility. According to the LAP all phenomena are able to move in any direction of time, as in the example of Newton's law, able to travel both forward and backward.

\section{The Principle of Conservation of Energy}

The word energy is reasonably new and its current sense is related to the principle of its conservation, established around $1847^{6}$. Not that an intuitive notion had not existed for some time, as we can see in the specialized literature (Lindsay, 1975). Atmospheric discharges, the force of the wind and rain, etc. were well known. However, these powers, as these forces of nature were called, were each considered distinct and unconnected. As we shall see, the principle of the conservation of energy would not only connect, but also unify them and fit them into the same manifestation of nature.

One of the key persons on the road to unification of the diverse views and manifestations of energy is undoubtedly Michael Faraday (1791-1867), who from an apprentice bookbinder, went on to become an extremely important figure in XIXth century physics. Coming from the poorest social classes of English society, he managed to get a job as an apprentice bookbinder which had a singular advantage, "There were many books there", as he revealed to a friend years later. Sometimes he spent the night alone reading by candlelight those books which most interested him. When he was twenty years old, a visitor to the workshop offered him a ticket for a series of conferences at the Royal Institution. The conferences were on electricity and the occult energies which must exist in nature. These not only awoke Faraday's interest but also pointed the way to a better life. But how was he to enter

\footnotetext{
${ }^{6}$ According to Atkins, ref. (Atkins, 2003), p.83, we owe the term “energy” to Thomas Young (1773-1829). While Robert Locqueneux, affirms that William Thomson introduced the term "energy”, this was in 1850. See (Locqueneux, 1996).
} 
academic life, if he had not even attended what we now call secondary school? Furthermore, his material resources were practically null. His father was a blacksmith and had never managed to give him anything.

Faraday then made use of the resource which he best dominated, the art of the bookbinder. He gathered the notes on Davy's conference then drew and inserted some designs of the equipment used in the demonstrations, using leather and engraving tools he bound all the material and sent it to Humphry Davy (1778-1829). After this Davy expressed a desire to know him, when they finally met Faraday was hired as a laboratory assistant. It was to take some years before he and Davy established a natural professional relationship.

At that time, Davy asked Faraday to study and try to understand a discovery that had been made in Denmark and was causing enormous repercussions throughout Europe. This was the experiment by Oersted (1757-1851). The phenomena of electricity and magnetism were known but completely unrelated. Then a lecturer in Copenhagen (Oersted) discovered that when passing an electric current through a wire it deflected the needle of a compass placed over it ${ }^{7}$.

Nobody had been able to explain how this had happened. According to some biographers of Faraday, unusually, his want of a better education curiously favoured him. His lack of formal studies in mathematics and physics prevented him from understanding the complexity of the physics problem being studied. Under these conditions Faraday began to study the relationships between electricity and magnetism in the summer of 1821. In the laboratory he fixed a magnet and imagined various invisible circular lines passing around it. If this were true, a loosely suspended wire could be dragged around, attracted by the invisible circles. He connected the battery and the wire behaved exactly as he had imagined.

Evidently, there are various hypotheses about Faraday's beliefs, in the sense of trying to explain the reason for his suppositions. Let us overlook these and simply point out that it was in this way that he made the discovery of the century. With the motion of a wire conducting electricity in a magnetic field he had discovered the working principle of the electric motor. As well as this discovery, Faraday managed to link electrical energy with magnetism and, although neither the concept of energy nor its conservation had yet been established, he made an enormous contribution to this by making a connection between electricity and magnetism. Later, Ampère (1775-1836), Gauss (1777-1855) and Ohm (1787-1854) developed theories which would allow them to comprehend profoundly the phenomena of the magnetic fields produced by electric currents and how they flow through conductors ${ }^{8}$.

The discovery of laws which linked the phenomena of electricity and magnetism created favourable conditions on the scientific plane for the Industrial Revolution to reach its second stage. As we know, the first stage of the Industrial Revolution was characterised by use of the steam engine and of coal as a form of energy which replaced water driven machines. The second stage of the Industrial Revolution was the era of electricity and its application in motors and electrical equipment, as well as new forms of transport with the automobile driven by the internal combustion engine. These changes in the means of production had enormous social implications which it is not appropriate to deal with here, even superficially.

In the period we are analysing, that is the end of the XVIIIth century until the early decades of the XIXth century, the physical sciences went through unprecedented transformations in terms of rupture from the Newtonian paradigm, as well opening up promising perspectives with new syntheses and theories explaining physical phenomena linked to light, heat, electricity and magnetism. There follows a synthesis of the most significant events:

1) Laplace (1749-1827) and his followers had formulated a mathematical theory based on Newtonian mechanics and which would be extended to thermal and optic phenomena. However, this theory had to be replaced by new developments in these areas in the decade 1815-25. Despite this, mathematization and the unifying proposal

\footnotetext{
${ }^{7}$ Maxwell, in his introduction to Faraday's, book reference (Faraday, 2003), describes the discovery of the phenomenon of the induction of electric currents by magnetic fields. "In December of 1824, Faraday had tried to obtain an electric current by means of a magnet. On three occasions, he made three fruitless complex attempts to produce a current in a wire by means of a current in another wire, or by means of a magnet. Even so, he persevered. On $12^{\text {th }}$ August 1831 he obtained the first proof that an electric current could induce another current in a different circuit... This was his first successful experiment. During a further nine days of experimenting, he reached the results described in his first series of 'Experimental Researches', read before the Royal Society on $24^{\text {th }}$ December 1831 ”.

${ }^{8}$ In September of 1820, in the Academy of Science session following that in which Oersted's experiments were announced in France, André-Marie Ampère published his first observations on the magnetic actions of electric currents. He showed the Academy that electric currents mutually attracted and repelled each other and followed those discovered laws which he called electrodynamics and which were fundamentally important for the elimination of magnetic fluids from science. Georg Simeon Ohm, began his experiments with electric currents in 1825. He used Volta's battery and later replaced it with copper-zinc thermoelectric elements, and could in this way establish the famous law which carries his name. See (Taton, 1995), pp. 210-215.
} 
of the physical world contained in the called Laplacian project had an important effect on the later development of physical theory ${ }^{9}$.

2) The publication in 1822 of the mathematical theory of heat by Joseph Fourier (1768-1830) brought the study of heat under mathematical analysis, previously applied only to mechanical problems. There was then established a difference between mathematical representation and physical representation. In 1840, influenced by the mathematical analogy between Fourier's heat theory and the theory of electrostatic, William Thompson explained these analogies on one side and on the other the laws of heat and electricity, introducing new views on the mechanics of particles in fluid and an elastic medium ${ }^{10}$.

3) In 1824, Sadi Carnot wrote a revolutionary work, "Reflections on the Driving Power of Fire”, in an industrial context in which the technology of steam engines was of growing interest among French and English engineers. As we can see in more detail in the studies dedicated to Lazare Carnot, the model used by Sadi to analyse the principles governing thermal engines owes much to his father, Lazare. Sadi's work ushered in a new science, thermodynamics, initially as a science of machines and later as a general science of transformation processes in nature ${ }^{11}$.

4) The theory of light waves of Augustin Jean Fresnel (1788-1827), which proposed that light propagates by vibrations of mechanical ether, brought optics into the conceptual framework of the mechanical view of nature. In 1830 this wave theory was accepted and physicists started to explore the great variety of physical and mathematical theories in an attempt to construct a coherent mechanical theory of optics ${ }^{12}$.

5) In 1827, while examining microscopic grains of pollen suspended in water, the Scottish botanist Robert Brown (1773-1858) noticed that the particles kept moving at random. This phenomenon, observable in both liquids and gases, was given the name Brownian motion. Even though this discovery is not directly related to the rifts we are dealing with, it is important that this fact be registered here, since it is directly linked to another important breach which was to occur more recently with atomic theory ${ }^{13}$.

6) Although formulation of the law of conservation of energy around 1847 had shaken the unity of physics-if this unity was seen within the above conceptual framework - it had nevertheless, placed the phenomena of heat, light and electromagnetism within the same basis of principles.

A fundamental step in the establishment of the conservation of energy principle was the measure of equivalence between mechanical work and heat. In the XVIIth and XVIIIth centuries physicists considered energy losses inside mechanical systems as isolated facts arising from non-mechanical processes and so never ventured to formulate a theory of equivalence between heat and mechanical energy. The concept of the conservation of mechanical energy had already been approached by some writers in the XVIIIth century. As we saw earlier, Leibniz formulated the principle of the conservation of vis viva. He had affirmed that vis viva, that is, the product of mass times velocity squared, is conserved in the mechanical process.

What must be observed with caution is that the use of the principle of conservation of vis viva, despite wide acceptance, did not signify a commitment to Leibniz's theories of nature. This principle was especially applied to

\footnotetext{
${ }^{9}$ For a more detailed study about Laplacian project and its implications on further developments of physics see (Oliveira, 2013).

${ }^{10}$ Joseph Fourier could be considered the first typical mathematician-physicist. His studies on heat propagation date from 1807, or earlier, and were gathered in a paper presented to the Academy of Science in 1811. His work "Théorie analytique de la chaleur", was published in 1822. In the solution to his famous equation on partial second order derivatives he presents the development of Fourier's series. See (Fourier, 1988).

${ }^{11}$ Sadi Carnot was fully aware of the importance of the changes that the development of the steam engine and its employment in diverse branches of the economy would bring for society. In the first pages of his celebrated essay, we read: "The study of these engines is of the greatest interest, their importance is enormous, their use is continually increasing, and they seem destined to produce a great revolution in the civilized world. Already the steam-engine works our mines, impels our ships, excavates our ports and our rivers, forges iron, fashions wood, grinds grain, spins and weaves our cloths, transports the heaviest burdens, etc. It appears that it must some day serve as a universal motor, and be substituted for animal power, water-falls, and air currents. Over the first of these engines it has the advantage of economy; over the other two, the inestimable advantage of power which may be employed at any time and in every place, and will never suffer an interruption in its work". See (Carnot, 1990), p. 2.

${ }^{12}$ Frensel's wave theory was a decisive contribution for the abandonment of the imponderable fluids theory. The origin of his work lies in the opposition not only to the scheme of imponderable fluids, but also to Laplace's corpuscular theory of light and the caloric theory for heat. At the start of these works, around 1814, Fresnel wrote that he suspected that light and heat were in some way connected with the vibrations of a fluid. His commitment to the concept of light as a kind of motion of a medium was basic for his optics theory in terms of the motion of wave propagation in a medium, luminisferous ether. See (Harman, 1982), p. 21.

${ }^{13}$ Einstein, in one of five articles published in 1905, would study Brownian motion and define a diffusion coefficient for particles in suspension, on the basis of the dimension of the particles, the viscosity of the fluid, its temperature and the Avogadro number. Following the inverse route, that is, having a way to measure this diffusion coefficient, it is possible to experimentally determine the Avogadro number. See Revue du CNRS, 2002, pp. 26-27.
} 
the perfectly elastic collision of bodies.

Jean Bernoulli is responsible for the more systematic and consistent accounts on the matter between 1720 and 1730. It is quite true that both he and Leibniz had already perceived that there could be losses in these collisions and they made an analogy between elastic collisions and springs which were prevented from expanding after compression. In this way, they explained the possible losses in energy consumption in the compression of bodies.

Daniel Bernoulli later discussed the operation of a thermal engine which utilised vis viva stored inside coal, by the generation of gases from the coal, but did not suggest any equivalence between heat and mechanical work. Nor did he attribute mechanical losses of the vis viva in inelastic collisions to the heat, as did his predecessors. He continued to consider the losses strictly from the mechanical point of view (Bernoulli, 1968).

At the beginning of the XIXth century, the measurement of vis viva through mechanical work, that is, the product of the force times distance, was introduced in various writings of a more technological stamp. This was the case of Carnot. Peter Ewart (1767-1842) who found a quantitative relationship between the heat generated by burning coal and the mechanical power or force which it could produce.

Lazare Carnot used the conservation of vis viva in mechanical work, although he did not adopt the term work for the product of the force and displacement. It was clear that the work was a measure of energy in the field of mechanics, since the sum of these quantities was conserved and if they could be summed up this meant that they were equivalents (Gillispie \& Youschkevitch, 1975). However, Gaspard-Gustave de Coriolis (1792-1843) in his famous book Du Calcul de l'Effet des Machines, published in 1829 corrected the expression for kinetic energy, introducing the constant 0.5 in the old expression for vis viva and adopted the term work and thus replacing the previous nomenclature (Coriolis, 1829).

Rumford's experiments (1798) on the heat produced in the machining of cannons showed that a great amount of heat was generated in this process. He then interpreted the phenomenon as arising from motion, that is, from friction, a point of view adopted by Humphry Davy and Ampère. Rumford also obtained a measurement of the mechanical equivalent of the heat. This established that one calory was equal to $570 \mathrm{~kg}-\mathrm{m}$, with an error of approximately $25 \%$.

The work of James Prescott Joule (1818-1889) in 1840 was fundamental in clarifying the conversion processes, as well as providing experimental confirmation of this quantitative equivalence between heat and mechanical work. At the start of the 40s of the XIXth century, Joule's investigations concentrated on the improvement of electric machines and the electrochemical field of investigation already embraced by Faraday as we have seen ${ }^{14}$.

Davy and Faraday had sought to formulate an electrical theory of chemical affinities and Joule also tried to elaborate this theory in the sense of unifying electrical, chemical and thermal phenomena, demonstrating their inter-conversion in the form of quantitative equivalence. In order to arrive at a relationship of this nature between work and heat, he built an electric machine in which mechanical work generated an electric current which, in turn, generated heat; the mechanism provided a numeric relationship between heat and mechanical work to be calculated. Joule soon concluded that mechanical work could be directly transformed into heat by friction.

In 1847, Joule illustrated the direct relationship between mechanical work and heat, in sketches which showed ropes connected to a weight, in such a way that the weight could be raised or lowered according to an increase or decrease in temperature. He did not publish these sketches of motion being transformed into heat, but simply affirmed that the heat was measured through the vis viva and consequently, that particles of the heated bodies were in motion ${ }^{15}$.

If Joule had a fundamental importance in establishing the principle of energy conservation, many others also played a part in this complex process, described in detail by Thomas Kuhn in his "Essential Tension", first published in English in 1977. Right at the beginning Kuhn affirms: Between 1842 and 1847, four scientists across Europe, Mayer, Joule, Colding and Helmholtz and, save for the latter, each ignorant of the others work, made

\footnotetext{
${ }^{14}$ Joule's first works concentrated on the perfection of electromagnets, the manufacture of galvanometers and on the properties of voltaic currents. After 1841 he concerned himself with the heat generated by electrical circuits and by electromagnetic machines. It was this work which led to his famous law.

${ }^{15}$ The experimental procedure adopted by Joule consisted in repeating twenty times the process of agitating a liquid by the movement of weights and measuring the final temperature of the agitated liquid. The walls of the recipient containing the liquid were hermetic and made of very thick wood, suitably treated to minimize any heat loss through convection or radiation. His conclusions were as follows: 1) the amount of heat generated by the friction of bodies, whether liquid or solid, is always proportional to the quantity of mechanical work expended; 2) the amount of heat capable of raising the temperature of 1 pound of water (weighed in a vacuum at a temperature between $55^{\circ} \mathrm{F}$ and $60^{\circ} \mathrm{F}$ ) by $1^{\circ} \mathrm{F}$ requires for its evolution the expenditure of a mechanical force required by the fall of 772 pounds (350.18 kg) through the space of one foot $(30.48 \mathrm{~cm})$. Between 1845 and 1847, Joule repeated these experiments using water, whale oil and mercury, obtaining for these compounds the mechanical equivalents equal to $781.5 \mathrm{lb} ; 82.1 \mathrm{lb}$ and $786.6 \mathrm{lb}$, respectively. See (Colin, 2003$)$, p. 31.
} 
public the hypothesis of the conservation of energy. Sadi Carnot, before 1832, Marc Séguin in 1839, Karl Holtzman in 1845 and G. Hirn in 1854, had each written, separately, of their conviction that heat and work are quantitatively equivalent and calculated a conversion coefficient or an equivalent.

What Kuhn's book proposes to show is a typical case of simultaneous discovery in science and its antecedents, the above citation providing the introductory elements. In addition, he also proposes to explain why, between 1830 and 1850; so many experiments and concepts necessary for the complete formulation of energy conservation arose to the surface of scientific consciousness. The analysis which Kuhn (Kuhn, 1977) makes in his famous article, enumerates three factors which most influenced this simultaneous discovery, namely:

1) availability of conversion processes;

2) interest in machines;

3) philosophy of nature.

The first factor concerns the development of science, the second the process of the Industrial Revolution and finally the third, the ideas and theories of physics at the time. Let us now look at these factors in more detail.

The availability of the conversion processes occurred due to a set of discoveries which began with the invention of the electric battery in 1800 by Alessandro Volta (1745-1827). According to the theory of Luigi Galvani (1737-1798), which was then dominant in France and England, an electrical current could be obtained from the forces expended by chemical affinity, and this conversion proved to be just one link in a much more complex chain which would in time become clearer. Also, as we have already mentioned, Faraday's work, the Oersted experiment and the magnetic properties which could produce motion—something known since antiquity—as well as the fact that it was possible to produce electricity by friction, were all systematically coming together and, little by little, the diverse connections between these phenomena were becoming known.

Furthermore, new experiments and phenomena were yet to be revealed. In 1822, Thomas Johann Seebeck (1770-1831) demonstrated that heat applied to a bimetallic belt produced directly an electric current. Twelve years later, Peltier managed to show the inverse process, namely, that an electric current could absorb heat and in this way produce cold. Within this more complex framework showed by Kuhn, Faraday's discoveries were only one more expansion of the field of phenomena in which conversion processes occurred.

Kuhn also called attention to a fact we consider very important for this undertaking, which is the role played by the principle of conservation of vis viva for the establishment of the principle of the conservation of energy. He affirms:

In most histories or pre-histories on the conservation of energy, it is supposed that the mode used to quantify the conservation processes was the dynamic theorem known since early XIXth century as the conservation of "vis viva". This theorem played an outstanding role in the history of dynamics, and proved to be a special case of conservation of energy. For that reason, it could well have become a model. Nevertheless, I believe that the prevailing impression that this was the case is erroneous. Conservation of the vis viva was important for Helmholtz to deduce the conservation of energy and a special case - that of free fall-from the same dynamic theorem which so helped Mayer. However, these personages also extracted important elements from another distinct tradition - that of water, wind and steam engineering — and this tradition is all important for the work of the other five precursors who attained a quantitative version of the conservation of energy (Kuhn, 1996).

With this citation, Kuhn reinforces one of the viewpoints we have adopted in this study, which is the importance of the expansion of machinery in society and its effects, one of the most notable being the development of general theories for the study of machines, it is not by chance that the second factor considered by him is the interest in machines. Kuhn also advances some commentaries about Lazare Carnot:

Until 1782, in "Essai sur les Machines en General", by Lazare Carnot, the product of the force times the distance did not receive a special name nor a conceptual priority within the dynamic theory. This new dynamic conception of the work concept was not really developed or widespread before 1819-39, when it was fully expressed in the works of Navier, Coriolis, Poncelet and others. All these works are concerned with the analysis of machines in motion. Consequently, work - the whole of force with respect to the distance (product of a force and a distance)-is its fundamental conceptual parameter. Among other significant characteristic results of this reformulation were: the introduction of the term "work" and of the units to measure it; the redefinition of "vis viva" as $0.5 \mathrm{mv}^{2}$ or $\mathrm{mv}^{2} / 2$ to conserve the conceptual priority of the measurement of work; and the explicit formulation of the law of conservation of equality of the work performed and the kinetic energy produced. Only when it was so reformulated, would the conservation of "vis viva" constitute a convenient conceptual model to quantify conversion processes, although almost none of the pioneers used it (Kuhn, 1996). 
Still following Kuhn's article, we see in general lines the ideas which guided the philosophy of nature of those who discovered the conservation of energy principle. The philosophical current denominated Naturphilosophie (Gusdorf, 1985) is of central importance and prime influence for many of those scientists who contributed to the establishment of the principle of the conservation of energy. The word Naturphilosophie comes from German Romanticism and expresses a concept which has no linguistic equivalent in French or English. Historians prefer to use the original term in order to designate a specific manner of speculation on the cultural plane where it had a rapid fecund development. It first appeared at the end of the XVIIIth century and in the 30s and 40s of the XIXth century when this kind of thinking was quite strong in German universities.

Kuhn describes the influence of Naturphilosophie on the discoverers of the principle of the conservation of energy, thus: Using the organism as the fundamental metaphor of their universal science, the Naturphilosophers constantly sought a single principle which would unify all natural phenomena. Friedrich Wilhelm Joseph Schelling (1775-1854), for example, sustained that magnetic, electrical, chemical and even organic phenomena must be interwoven, forming a great fusion... [which] would include the whole of nature ${ }^{16}$.

Schelling insistently sought the processes of conversion and transformation in the science of his time. Many of his followers dominated teaching in German universities during the first third of the XIXth century, presenting the new conversion phenomena in a way similar to Schelling's.

It is well known that Oersted was a Naturphilosoph, since he persistently pursued the idea of a relationship between electricity and magnetism, driven mainly by his philosophical convictions. In short, many of the scientists who worked in the laboratories of process conversion extracted from their experiments a conception of the physical processes. If we wish to establish connections of other personages with Naturphilosophie, we could add that Colding was closely linked with Oersted. Liebig studied for two years with Schelling. Adolf Hirn (1815-1890) frequently cited both Lorenz Oken (1779-1851) and Kant. Robert Mayer did not study Naturphilosophie, however, he had close friends linked to these studies. The father of Helmholtz, friend of Johann Fichte (1762-1814), younger than him, was an adept of that philosophy and exhorted his son to abandon strict mechanicism.

As we see, contrary to what positivists may think, metaphysics is not only useful in the formulation of scientific theories, but it is also perhaps inevitable that ideas of a purely metaphysical origin may influence scientific thinking. There are numerous examples. As well as this case-the establishment of a fundamental principle of physics - we have many others, like the universal law of gravitation.

Closing these considerations on the principle of the conservation of energy, we should observe that if the ideas on the unification of natural phenomena coming from Naturphilosophie, on the one hand played a fundamental role in this new synthesis in physics, uniting diverse phenomena from different fields into a more general principle, on the other, this discovery also fomented new conceptions and strengthened a dynamistic view of nature. In reality, this was the subjugation of the Newtonian paradigm which was spreading through diverse fields of physics, very often with the use of implements developed by Newton himself. This is the case, for example, of AndréMarie Ampère (1775-1836), who affected the first physical-mathematical synthesis between electricity and magnetism in his Memoire sur la Théorie Mathématique des Phénomènes Électrodinamiques Uniquement Déduite de l'Expérience, published in 1827.

\section{Final Remarks and Conclusion}

The conservation of energy is a common characteristic in many physical theories. From the historical point of view it is a consequence of the development of physical sciences of XIXth century in spite of its restrict use in machines context by Lazare Carnot in XVIIIth century. From a mathematical point of view some elegant proofs appear as Noether's theorem. Emmy Noether's (1882-1935) first theorem states that any differentiable symmetry of the action of a physical system has a corresponding conservation law. This was proved in 1915 and published in 1918. The action in a physical system is defined as the integral over time of a Lagrangian function, from which the system's behaviour can be determined by the principle of least action.

With the development of physics in XXth century the principle of conservation of energy acquires some other features. The discovery of special relativity by Albert Einstein (1879-1955), energy was proposed to be one component of an energy-momentum 4-vector. Each of the four components, one of energy and three of momentum, of this vector is separately conserved along the time, in any closed system, as seen from any given inertial

\footnotetext{
${ }^{16}$ It is common to consider the influence of Immanuel Kant (1724-1804) and even that of Leibniz and dynamism on Schelling. For the first two thinkers, the first concept is the inherent force of matter.
} 
reference frame.

In quantum mechanics, energy of a quantum system is described by a self-adjoint (Hermite) operator called Hamiltonian, which acts on the Hilbert space, a space of wave functions, of the system. If the Hamiltonian is a time independent operator, emergence probability of the measurement result does not change in time over the evolution of the system. Thus, the expectation value of energy is also time independent. The local energy conservation in quantum field theory is ensured by the quantum Noether's theorem for energy-momentum tensor operator. This means that the conservation of energy in time is a well-defined concept even in quantum mechanics.

\section{References}

Atkins, P. (2003). Galileo's Finger. UK: Oxford University Press.

Bernoulli, D. (1968). Hydrodynamics. New York: Dover Publication, Inc.

Carnot, S. (1990). Réflexions sur la Puissance Motrice du Feu. Paris: Éditions Jacques Gabay.

Colin, L. G. (2003). From the Steam Machine to Absolute Zero. Mexico: Fondo de Cultura Editors.

Coriolis, G. G. (1829). Du Calcul de l'Effet des Machines. Paris: Carilian-Goeury, Librairie.

De Maupertuis, P. L. M. (1744). Accord des Différents Lois de la Nature qui Avaient Jusqu'ici Paru Incompatibles, Memoires de l'Academie des Sciences de Paris.

Dugas, R. (1988). A History of Mechanics. New York: Dover Publications, Inc.

Euler, L. (1952). Methodus Inveniendi Lineas Curvas Maximi Minimive Proprietates Gaudentes (Vol. XXIV). Lausanne: Leonhardi Euleri Opera Omnia, s. I.

Faraday, M. (2003). A Chemical History of a Candle. Rio de Janeiro: Contraponto Editors.

Fourier, J. B. P. (1988). Théorie Analytique de la Chaleur. Paris: Éditions Jacques Gabay.

Gueroult, M. (1967). Leibniz, Dynamique et Metaphisique. Paris: Aubier Editions Montaigne.

Gillispie, C. C., \& Youschkevitch, A. P. (1979). Lazare Carnot Savant et sa Contribuition a la Théorie de l'Infinie Mathematique. Paris: Librairie Philosophique J. Vrin.

Gusdorf, G. (1985). Le Savoir Romantique de la Nature. Paris: Payot.

Harman, P. M. (1982). Energy, Force and Matter: The Conceptual Development of Nineteenth-Century Physics. Cambridge University Press. http://dx.doi.org/10.1017/CBO9780511665394

Kuhn, T. S. (1996). The Essentian Tension. Mexico: Fondo de Cultura Editors.

Lagrange, J. L. (1989). Mécanique Analytique. Paris: Éditins Jacques Gabay.

Locqueneux, R. (1996). Prehistoire \& Histoire de la Thermodinamique Classique, Une Histoire de la Chaleur, no. 45. Paris: Librairie A. Blanchard.

Lindsay, R. B. (1975). The Concept of Energy and its Early Historical Development (p. 12). Pennsylvania: Brown University.

Moreira, I. C. (1998). Maupertuis and the Least Action Principle. Rio de Janeiro: Federal University Publication.

Newton, I. (1952). Mathematical Principles of Natural Philosophy. London: Great Books of the Western World.

Oliveira, A. R. E. (2013). A History of the Work Concept: From Physics to Economics. Netherlands: Springer.

Oliveira, A. R. E. (2012). The Concept of Work in the Development of Applied Mechanics: Carnot and Coriolis. Invited talk in $32^{\text {nd }}$ International Congress of the Italian Society of Historians of Physics and Astronomy, Rome.

Plank, M. (1993). A Survey of Physical Theory. New York: Dover Publication, Inc.

Truesdell, C. (1980). The Tragicomical History of Thermodynamics 1822-1854. New York: Springer-Verlag. http://dx.doi.org/10.1007/978-1-4613-9444-0 